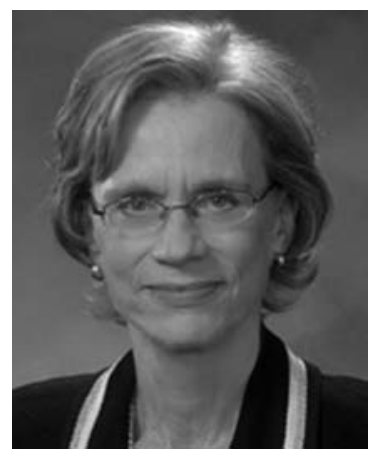

\title{
A Conversation with Ann Graybiel
}

\author{
INTERVIEWER: KAREN CARNIOL \\ Deputy Editor, Cell
}

Ann Graybiel is an Institute Professor at Massachusetts Institute of Technology.

Karen Carniol: One of your research directions is about value judgments and decision-making in terms of potential trade-offs or rewards or outcomes.

Dr. Graybiel: In everyday life, we all face situations in which our options are mixed-if we do something, the outcome will be partly good and partly bad. This kind of decision-making is called approach-avoidance decisionmaking, because we have to decide whether to go ahead and do something or to avoid doing it. We face a conflict between approach motivation and avoidance motivation. There are formal ways to test such approach-avoidance conflict, and these tests have been helpful in the diagnosis of problems related to anxiety and depression. Some years ago, Ken-ichi Amemori came to our lab, and he worked out a way that we could use this kind of conflict task in macaque monkeys. This opened up the chance to look at the neural circuits underlying what we think is emotion-related decision-making.

We had been trying to figure out what certain corticostriatal pathways do functionally, and had guessed they might have something to do with weighing different levels of goodness and badness in a given situation. We're interested in compartmental ordering in the striatum. This is a large region deep in the brain that is sort of like a huge ball of cells, but threading through it, like the trains in driftwood, are specialized cellular zones that we thought might be related to something about reinforcement or emotion.

In the search for what those regions might do, Ken-ichi Amemori and I targeted regions of the neocortex that we knew projected to these specialized striatal zones-the so-called "striosomes" or striatal bodies. These cortical regions have been implicated in conditions related to mood disorders such as OCD, addiction, and anxiety disorders. We set out to find out whether we could change the macaques' approach-avoidance decision-making in a task modeled on the tasks used with human subjects by manipulating activity in regions of the anterior cingulate cortex and caudal orbitofrontal cortex, both prefrontal regions that we had found to project to striosomes and that seemed to correspond to cortical regions picked out by a number of human neural imaging studies as abnormal in neuropsychiatric disorders.

We hope this work will have translation value but there's a very deep basic science underpinning it.

The deepest is how does a brain work, and how is it that we have a giant forebrain and a huge neocortex. Second only to the neocortex in the so-called progression index of brain evolution is this giant striatum, the giant ball of cells underneath the neocortex. We know that this deep-brain system communicates with the neocortex in elaborate loop circuits, but in the old days, it was thought to have its primary effects downstream, by way of descending pathways leading to premotor regions that are central pattern generators for movement-regions that control functions like walking and breathing.

Out of this basic anatomy, we developed the idea that maybe the reason the basal ganglia in primates and some other species have such strong output to the neocortex (through a series of steps) is that the basal ganglia loops help us to make new patterns, even cognitive patterns, that we can rely on just as we rely on the brainstem and spinal motor pattern generators for breathing and other elemental activities.

Karen Carniol: Going back to the manipulations, microstimulations in the anterior cingulate cortex that could shift the trade-off values in the macaques, where do you think the projections were that were causing that?

Dr. Graybiel: We found many years ago that those regions, the anterior cingulate cortex and, we think, the caudal orbitofrontal cortex, preferentially project to these distributed compartments in the striatum called striosomes. Dr. Satoko Amemori in our lab did the interesting anatomical tracing of the connections in the very animals in which we did the recording and the microstimulation and found that the region that we manipulated to change the decision-making of the monkeys probably projects striosomes preferentially. This is a recent observation, and we are still doing more experiments to confirm this. 
The pathway she works on seems to be related to some balance between pessimism and optimism.

The reason that we are working so hard on this is that maybe this is a clue to how the basal ganglia can affect emotion. Basal ganglia-related pathways definitely are related to whether we can move or not, as witnessed by the problems of patients with Parkinson's disease. But in addition, there's another side of basal ganglia function that somehow relates to a more cognitive or even emotional side of things. Here we are trying to find out whether the striosomes, dispersed though much of the striatum, are functionally connected with regions of the brain that we can relate to mood and emotion. Maybe they help decide what we're motivated to do or not motivated to do. This is such a basic function that it is conserved through evolution, and in fact, the striatum and its striosome-related pathways are too.

Karen Carniol: You talked about shifting the macaques towards more pessimistic decision-making by the microstimulation. How could that interplay with anxiety?

Dr. Graybiel: I don't know whether there really is a "worry circuit," but we do think that anxiety is likely involved in the results. One reason is that when we do this microstimulation, and induce the monkeys to have more pessimistic decision-making, we can completely reverse this effect by giving anxiety-reducing drugs such as diazepam, and the effect is dose-dependent.

Karen Carniol: Another area of your research is how behaviors turn into habits.

Dr. Graybiel: We have been startled to find that that by using optogenetic manipulations of tiny parts of the neocortex of rodents, we can prevent a habit from forming or block a habit that has been formed and then bring it back.

We first found that there is remarkable plasticity in the activity of neurons in the striatum and neocortex as the animals learn habits. We put many electrodes in the sensorimotor part of the striatum in rats and mice, and trained them to run in a T-maze. It turns out that over time, as they learn a habit, the cells become more and more active at the beginning and end of maze runs, but less and less active during the runs. It was as though the brain regions were forming activity markers for the habit as a wholethe entire sequence making up the habit. Then we found that in the nonsensorimotor part of the striatum, it isn't like that at all! Neurons there like to concentrate on the decision-making period of the task. When the animal gets to the $\mathrm{T}$ in a $\mathrm{T}$-maze, the activity of those neurons swells up, presumably in relation to the animal's deciding. Then when that behavior becomes a habit, they stop firing as much. This taught us that different corticostriatal loops can be simultaneously active but can pay attention to quite different aspects of the behaviors being learned.

In psychology there is a longstanding view that decision-making circuits are recruited gradually as the animal learns to make the decisions. Then, as the animal makes the decisions over and over again, the brain can shift a behavior to a habit. This has the great advantage that then, in the habitual mode, we don't have to think out the decision every time. This sequencing of brain activity has been recognized to occur both in the striatum and in the neocortex. And we wondered whether it also had this sort of bracketing of learned, habitual behaviors. Kyle Smith came to our lab and recorded there, and we found that the task bracketing did form, at least in the upper layers of the cortex.

In those upper layers, the cell ensembles at first tended to fire throughout the time as the animals were learning. No great pattern was forming. But when that behavior really got stamped in, after a long training period, all of a sudden, we got the beginning-and-end pattern up in the cortex-very much like the pattern we had seen to develop in the sensorimotor striatum. So maybe we have to have both the striatum and the neocortex developing that pattern until the brain decides "This behavior is a keeper." If it's a keeper, you have a habit.

It is that critical neocortical region that we turn on and off, optogenetically, to turn habits on and off. 


\section{$\$_{\text {CSH }}^{\infty}$ Cold Spring Harbor Symposia SYMPOSIA}

\section{A Conversation with Ann Graybiel}

Cold Spring Harb Symp Quant Biol 2014 79: 277-278

Access the most recent version at doi:10.1101/sqb.2014.79.11

\section{License}

Email Alerting Receive free email alerts when new articles cite this article - sign up in Service the box at the top right corner of the article or click here. 